\title{
Code and Manual
}

- Source code is archived at: https://zenodo.org/record/572819\#.WRCy7xiZORs (DOI: 10.5281/zenodo.572819). Link to the repository is provided in manuscripts.

The Plume-SPH code, together with a user manual providing instructions for installation, running and visualization are archived at https://zenodo.org/record/572819\#.WRCy7xiZORs (DOI: 10.5281/zenodo.572819). The input data for all simulations presented in this work are archived in the same repository. The MIT license governs the distribution and use of the code and associated documentation files. Permission is granted, free of charge, to any person to deal in the software without restriction. The complete copyright statement can be found in the repository : https://github.com/PlumeSPH/plume-sph/blob/master-1.0/COPYING.

- User manual and input data for test runs are also archived in the same repository.

- Output data of simulations presented in the paper is around tens of GB and is archived in UB-Box. Access will be provided to all upon request. 